\title{
The Strange Quark Polarisation from COMPASS data
}

\author{
O.Kouznetsov (on behalf of the COMPASS collaboration)
}

\author{
Joint Institute of Nuclear Research, Joliot Curie 6, 141980 Dubna, Russia \& \\ Universität Mainz, Institut für Kernphysik, 55099 Mainz, Germany
}

\begin{abstract}
The strange quark helicity distribution $\Delta s(x)$ was derived at $\mathrm{LO}$ from the inclusive asymmetry $A_{1, d}$ and the semi-inclusive asymmetries $A_{1, d}^{\pi+}, A_{1, d}^{\pi-}, A_{1, d}^{K+}, A_{1, d}^{K-}$ measured by COMPASS in polarised deep inelastic muon-deuteron scattering. The distribution of $\Delta s(x)$ is compatible with zero in the whole measured range. The value of the first moment of $\Delta s$ and its error are very sensitive to the assumed value of the ratio of the $\bar{s}$-quark to $u$-quark fragmentation functions into positive kaons $\int D_{\bar{s}}^{K^{+}}(z) d z / \int D_{u}^{K^{+}}(z) d z$.
\end{abstract}

Keywords: Nucleon Spin; DIS; Polarised PDF; Strange Quark Polarisation

PACS: 13.60.Hb; 13.60.Le; 13.88.+e; 24.85.+p

Among the various sea quarks contributing to the nucleon spin, the strange quarks are the only ones accessible in inclusive lepton-nucleon scattering experiments. The first moment of the strange quark helicity distribution, $\Delta s+\Delta \bar{s}$, has been found to be negative already twenty years ago in the EMC experiment [2]. This result has been confirmed with improved precision by recent measurements performed by HERMES [3] and by COMPASS [4] which has obtained

$$
\Delta s+\Delta \bar{s}=-0.09 \pm 0.01 \text { (stat.) } \pm 0.02 \text { (syst.) }
$$

at $Q^{2}=10(\mathrm{GeV} / c)^{2}$ at leading order (LO) in QCD. Inclusive experiments, however, provide an evaluation of the first moment $\Delta s+\Delta \bar{s}$ only. Direct information on the distribution $\Delta s(x)$ can be obtained from semi-inclusive channels such as charged kaon production. These measurements, which require particle identification, were performed by HERMES [5, 6] and COMPASS [7](present analysis).

In order to access the spin-dependent structure functions measurements of the inclusive and semi-inclusive double spin asymmetries were performed. The full deuteron statistics of COMPASS ${ }^{1}$ (years 2002-2004 and 2006) was used. The data cover the range $Q^{2}>1(\mathrm{GeV} / c)^{2}$ and $0.004<x<0.3$. Hadron identification was performed using the $\mathrm{RICH}$ detector in the momenta interval $10<p<50 \mathrm{GeV} / c$. An unfolding procedure to the samples of identified pions and kaons was applied to correct rates and asymmetries. It was found that the contamination of the samples has only a small effect on the asymmetries (Fig. 1). The results of HERMES, [5], are shown for comparison. The two sets of measurements are well compatible and in the region of kinematic overlap the statistical precision of the two experiments is generally comparable. However COMPASS

${ }^{1}$ A description of the spectrometer can be found in [1]. 


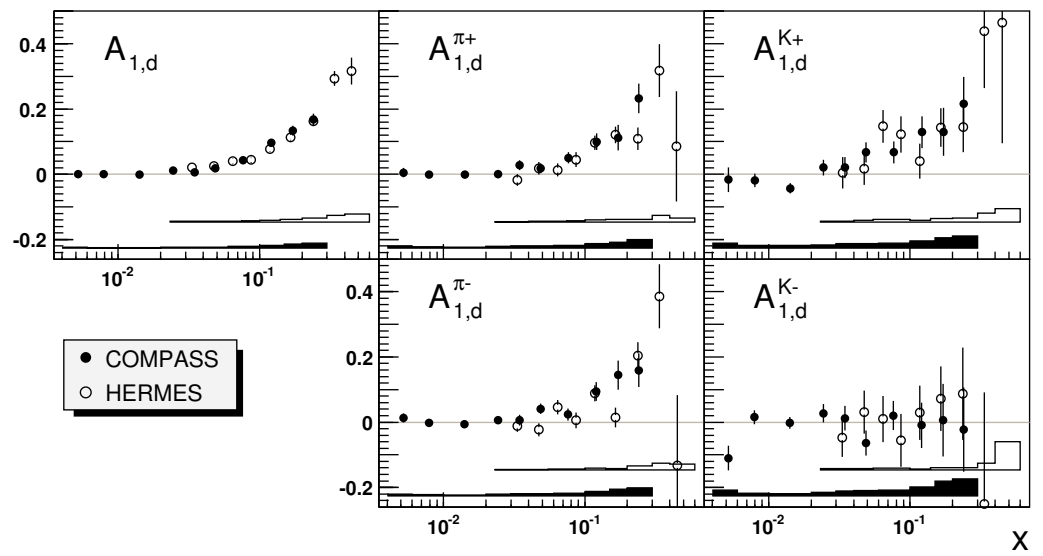

FIGURE 1. Comparison of spin asymmetries of COMPASS as a function of $x$ with results of HERMES [5]. Bands at bottom of graphs represent systematic uncertainties.

extends the measured region towards $x=0$ by an order of magnitude. It is also observed that all asymmetries, except $A_{1, d}^{K^{-}}$, are quite similar to each other. The $K^{-}$asymmetry is consistent with zero over the full range of $x$.

The polarised parton densities (PDFs) are evaluated at $Q_{0}^{2}=3(\mathrm{GeV} / c)^{2}$ in each bin of $x$ by a least square fit on the asymmetries assumed to be independent of $Q^{2}$. For the unpolarised distributions, $q\left(x, Q^{2}\right)$, LO MRST04 [9] are used. The two sets of fragmentation functions, $D_{q}^{h}\left(z, Q^{2}\right)$, are taken from the DSS [10] group and the EMC[11] measurements of the $u$ quark fragmentation into $\pi$ and $K$. The fitted polarised PDFs are shown in Fig. 2. The estimates of the truncated first moments $\Delta u_{v}+\Delta d_{v}, \Delta \bar{u}+\Delta \bar{d}$ and $\Delta s$ are given in Table 1 . The value quoted for the valence quarks is in good agreement with the one derived in our previous publication [4] from the difference asymmetries for nonidentified hadrons obtained from a partially overlapping data sample $(0.26 \pm 0.07 \pm 0.04$ for $x<0.3$ and $0.41 \pm 0.07 \pm 0.06$ for full $x$ interval at $\left.Q^{2}=10(\mathrm{GeV} / c)^{2}\right)$.

The dependence of $\Delta s(x)$ on the FFs can be further explored in relation with the charged kaon asymmetry $A_{1, d}^{K^{+}+K^{-}}$. This asymmetry is a weighted average of $A_{1, d}^{K^{+}}$and $A_{1, d}^{K^{-}}$with weights given by the spin-averaged $K^{+}$and $K^{-}$cross-sections. It is found to be very stable with respect to the ratio $\sigma^{K^{-}} / \sigma^{K^{+}}$. At LO, the cross-section ratio only depends on the unpolarised PDFs and on the ratios of unfavoured to favoured and strange to favoured FFs:

$$
R_{U F}=\frac{\int D_{d}^{K^{+}}(z) d z}{\int D_{u}^{K^{+}}(z) d z}, \quad R_{S F}=\frac{\int D_{\bar{s}}^{K^{+}}(z) d z}{\int D_{u}^{K^{+}}(z) d z}
$$

which are respectively equal to 0.13 and 6.6 for the DSS FFs at $Q^{2}=3(\mathrm{GeV} / c)^{2}(0.35$ and 3.4 for the EMC FFs). The values shown in Fig. 3-left have been obtained with the MRST PDFs and the DSS FFs. 


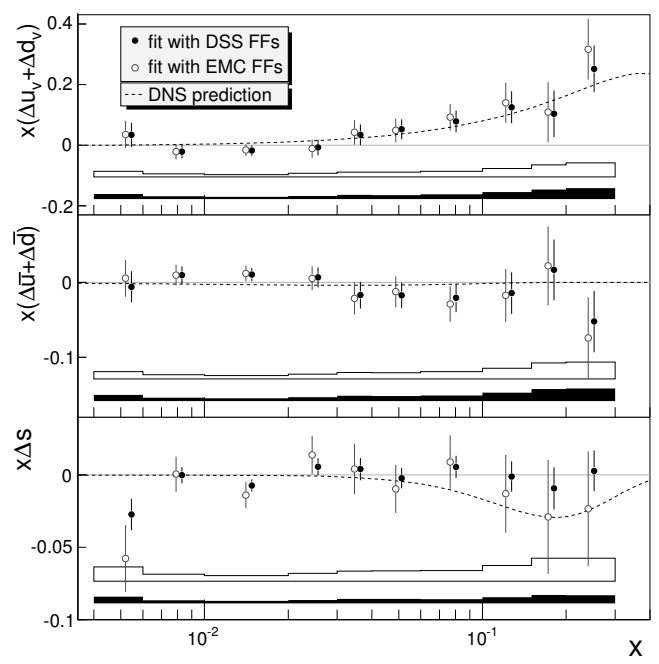

FIGURE 2. The quark helicity distributions evaluated at common value $Q^{2}=3(\mathrm{GeV} / c)^{2}$ as a function of $x$ for two sets of fragmentation functions (DSS and EMC). Bands at bottom of graphs represent systematic uncertainties. The curves represent the LO DNS parameterisation of polarised PDFs [8].

TABLE 1. First moments $\Delta u_{v}+\Delta d_{v}, \Delta \bar{u}+\Delta \bar{d}$ and $\Delta s$ from the COMPASS data and also from the DNS fit at LO [8] truncated to the range of the measurements $(0.004<x<0.3)$.

\begin{tabular}{|c|c|c|c|}
\hline \multicolumn{2}{|c|}{$\mathrm{FF}$} & DSS & EMC \\
\hline \multirow{2}{*}{$\Delta u_{v}+\Delta d_{v}$} & measur. & $0.26 \pm 0.06 \pm 0.02$ & $0.30 \pm 0.08 \pm 0.02$ \\
\hline & DNS & \multicolumn{2}{|c|}{0.225} \\
\hline \multirow{2}{*}{$\Delta \bar{u}+\Delta \bar{d}$} & measur. & $-0.04 \pm 0.03 \pm 0.01$ & $-0.05 \pm 0.04 \pm 0.01$ \\
\hline & DNS & \multicolumn{2}{|c|}{-0.009} \\
\hline \multirow{2}{*}{$\Delta s(=\Delta \bar{s})$} & measur. & $-0.01 \pm 0.01 \pm 0.02$ & $-0.05 \pm 0.03 \pm 0.03$ \\
\hline & DNS & \multicolumn{2}{|c|}{$\frac{1}{-0.03}$} \\
\hline
\end{tabular}

To take advantage of the similarity between $A_{1, d}^{K^{+}+K^{-}}$and $A_{1, d}$, it is convenient to write the strange quark polarisation $\Delta s / s$ in the form

$$
\frac{\Delta s}{s}=\frac{1}{\xi}\left[A_{1, d}+\left(A_{1, d}^{K^{+}+K^{-}}-A_{1, d}\right) \frac{Q / s+\alpha}{\alpha-0.8}\right]
$$

where $Q$ is the non-strange quarks density $Q=u+\bar{u}+d+\bar{d}, \alpha=\left(2 R_{U F}+2 R_{S F}\right) /(2+$ $\left.3 R_{U F}\right)$ and $\xi$ is a correction factor. The above formula shows that in the special case where $A_{1, d}^{K^{+}+K^{-}}$would be strictly equal to $A_{1, d}$, the strange quark helicity would become insensitive to FFs and its first moment would be small and positive $(\approx 0.006)$. Otherwise the main dependence on the FFs is due to $R_{S F}$, which appears only in the numerator of $\alpha$, and its effect is amplified by the large values of the ratio $Q / s$. Negative values of $\Delta s$ correspond to negative values of $A_{1, d}^{K^{+}+K^{-}}$at low $x$ where $A_{1}^{d} \approx 0$ or to $A_{1, d}^{K^{+}+K^{-}}<A_{1, d}$ at higher $x$. 

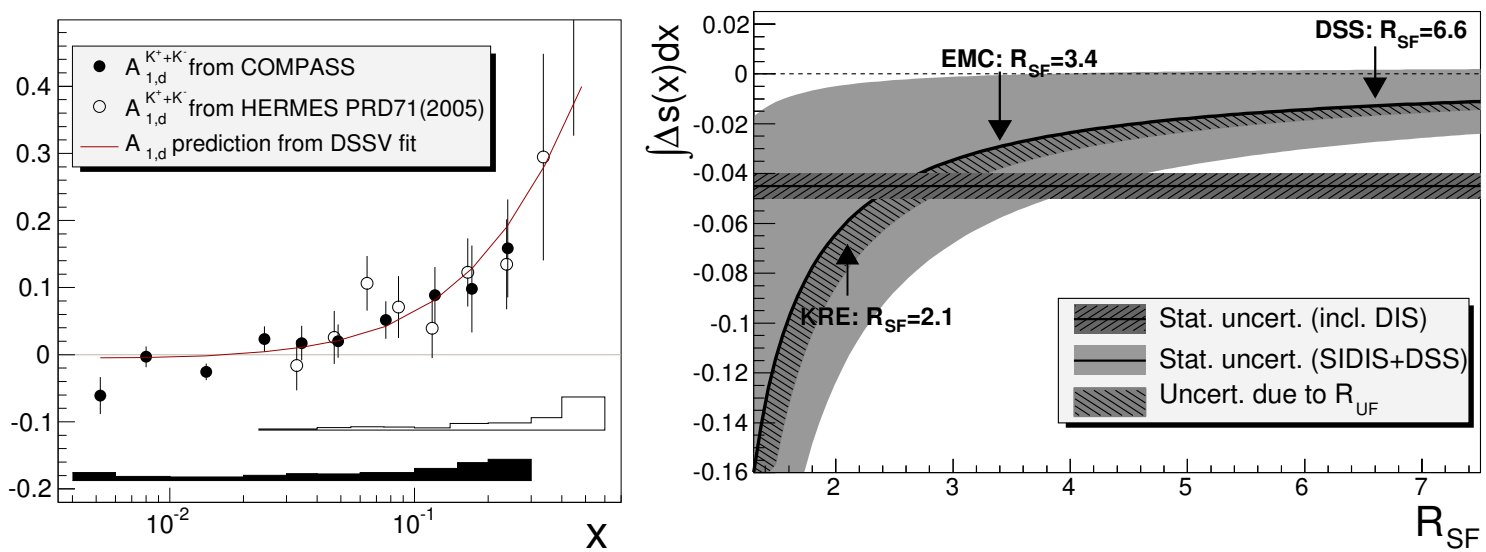

FIGURE 3. Left: Charged kaon asymmetries obtained with cross-section weights from MRST PDFs and DSS FFs. The curve shows the $A_{1, d}$ prediction of the DSSV fit [12]. Right: Integral of $\Delta s$ over the measured range of $x$, as a function of the ratio $R_{S F}$ for $R_{U F}$ fixed at the DSS value of 0.13 (thick solid curve). The light-grey area shows the statistical uncertainty and the hatched band inside of it shows the effect of increasing $R_{U F}$ to 0.35 (EMC value). The horizontal band represents the full moment of $\Delta s$ derived from the COMPASS value of the first moment of $g_{1}^{d}(x)$ (Eq. 1). The values of $R_{S F}$ corresponding to DSS[10], EMC[11] and KRE[13] parameterisations of FFs are indicated by arrows.

Fig. 3-right shows the variation of the first moment of $\Delta s$ truncated to the measured region as a function of $R_{S F}$. For $R_{S F} \geq 5$, we observe that the values are close to zero and larger than the full moment derived from the inclusive analysis (Eq. 1). The contribution from the region $x>0.3$ is limited by the positivity condition $|\Delta s(x)| \leq s(x)$ and cannot exceed 0.003 in absolute value. Thus any difference between the truncated SIDIS moment and the full moment (found to be negative from inclusive measurements) must be compensated by an unmeasured contribution in the low $x$ region. In particular this is the case for the DSS FFs where $R_{S F}=6.6$. The difference never exceeds two standard deviations, so that no firm conclusions can be drawn. In contrast, if $R_{S F} \leq 4$, the asymmetry $A_{1, d}^{K^{+}+K^{-}}$becomes less and less sensitive to $\Delta s$ because $D_{\bar{S}}^{K^{+}}$is small.

An extraction of FFs (first of all $R_{S F}$ ratio) from COMPASS data is in progress.

\section{REFERENCES}

1. P. Abbon, et al., Nucl.Instr. Meth. A 577, 455-518 (2007).

2. J. Ashman, et al., Phys. Lett. B 206, 364-380 (1988).

3. A. Airapetian, et al., Phys. Rev. D 75, 012007, 1-48 (2007).

4. M. Alekseev, et al., Phys. Lett. B 660, 458-465 (2008).

5. A. Airapetian, et al., Phys. Rev. D 71, 012003, 1-36 (2005).

6. A. Airapetian, et al., Phys. Lett. B 666, 446-450 (2008).

7. M. Alekseev, et al., CERN-PH-EP-2009-008 (2009), arXiv:0905. 2828 ( hep-ex).

8. D. de Florian, G. A. Navarro, and R. Sassot, Phys. Rev. D 71, 094018, 1-12 (2005).

9. A. Martin, W. Sterling, and R. Thorne, Phys. Lett. B 636, 259-264 (2006).

10. D. de Florian, R. Sassot, and M. Stratmann, Phys. Rev. D 75, 114010, 1-26 (2007).

11. A. Arneodo, et al., Nucl. Phys. B 321, 541-560 (1989).

12. D. de Florian, R. Sassot, M. Stratmann, and W. Vogelsang, Phys. Rev. Lett. 101, 1-4 (2008).

13. S. Kretzer, Phys. Rev. D 62, 054001, 1-13 (2000). 